\title{
AMP-activated protein kinase (AMPK) contributes to the anti-obesity effects and adipose tissue browning of alpha-lipoic acid in ovariectomized rats
}

Hsin-Hsueh Shen ( $\nabla$ hsshen@mail.ndmctsgh.edu.tw )

National Defense Medical Center https://orcid.org/0000-0002-5937-3472

Ming-Ting Chung

Chi Mei Medical Center

Shieh-Yang Huang

Kaohsiung Armed Forces General Hospital

Ching-Wen Kung

Tzu Chi University of Science and Technology

Shu-Ying Chen

Hung Kuang University: HungKuang University

\section{Yi-Wen Chen}

National Defense Medical Center

Pao-Yun Cheng

National Defense Medical Center

Yen-Mei Lee

National Defense Medical Center

\section{Research}

Keywords: a-lipoic acid, menopause, obesity, beige adipocyte, AMPK

Posted Date: March 26th, 2021

DOl: https://doi.org/10.21203/rs.3.rs-338317/v1

License: (1) (1) This work is licensed under a Creative Commons Attribution 4.0 International License. Read Full License 


\section{Abstract}

\section{Background}

Bilateral ovariectomy is an experimental model used to analyze the conditions of menopause and develop strategies for alleviation of the deleterious effects during estrogen deficiency. Brown and beige adipocytes exert thermogenesis capacities and are promising therapeutic strategy for obesity. This study aims to investigate the adipose tissue browning potentials of antioxidant a-lipoic acid (ALA) and underlying mechanisms involved in ovariectomized (Ovx) rats.

\section{Methods:}

Eight weeks old female Wistar rats were randomly divided into Sham or Ovx groups. The Ovx rats were subjected to bilateral ovariectomy and administered with ALA 200 or ALA $300 \mathrm{mg} / \mathrm{kg} / \mathrm{day}$ (gavage) for 8 weeks.

\section{Results:}

Ovx group significantly increased boy weight (BW) and fat pad mass as compared to Sham group, while ALA supplementation reversed these changes. Lipid profiles including serum triglycerides (TG), total (TC) and low-density lipoprotein cholesterol (LDL-C) levels were significantly elevated in the Ovx group, whereas the ALA treatment showed a significant decrease in these levels. Furthermore, high density lipoprotein cholesterol (HDL-C) and myokine irisin secretion were increased by ALA as well. Morphology results showed ALA treatment reduced Ovx-induced adipocyte hypertrophy and enhanced UCP1 expression by immunohistochemical staining in inguinal WAT. Protein expression of brown fat-specific markers UCP1, PRDM16 and CIDEA was markedly reduced in Ovx rats, whereas ALA treatment reversed these changes. ALA significantly increased liver kinase B1 (LKB1) and phosphorylation of AMP-activated protein kinase (AMPK) and the downstream acetyl-CoA carboxylase (ACC) that were decreased by Ovx, suggesting the browning effects were mediated by AMPK signaling.

\section{Conclusions:}

ALA ameliorates obesity caused by hormone deprivation in menopause via conversion of white to beige adipocytes concomitant with the activation of AMPK signaling.

\section{Introduction}

During menopause, women experience weight gain and re-deposition of body fat into the waist [1]. Obesity and fat accumulation increase the risk of metabolic syndromes and cardiovascular diseases, such as diabetes, dyslipidemia and hypertension [2]. Despite estrogen exerts beneficial effects against menopause associated metabolic abnormalities [3], long-term hormonal replacement therapy retains the limitations of adverse effect including breast and cervical cancer and is not primarily strategy for 
menopausal obesity and metabolic dysfunctions [4]. Seeking alternative therapy for menopauseassociated metabolic syndrome acquires further investigation.

In mammals, three types of adipose tissue have been identified based on the function and characteristics: white adipose tissue (WAT), brown adipose tissue (BAT), and beige adipose tissue [5]. WAT stores energy in the form of triglycerides and regulates whole-body energy homeostasis, while BAT dissipates energy as heat through enhanced mitochondrial content and uncoupling protein 1 (UCP1) [6]. Recently, recruitment of beige adipocytes has been observed in WAT in response to various stimuli such as cold exposure and pharmacological agents $[7,8]$.

AMP-activated protein kinase (AMPK) is a master regulator of energy homeostasis and activated by ATPdepleting conditions such as physical exercise, ischemia, and glucose deprivation [9]. AMPK is regulated

via phosphorylation of $\mathrm{Thr}^{172}$ by upstream kinases, liver kinase B1 (LKB1) or $\mathrm{Ca}^{2+} /$ calmodulin-dependent protein kinase kinases [10]. Activation of AMPK resulted in phosphorylation and inhibition of acetyl-CoA carboxylase (ACC), which plays an important role in fatty acid synthesis, and therefore promotes fatty acid oxidation as well as reduces fat accumulation [11]. In addition to the metabolic pathway, AMPK also regulates inflammatory cascades for improving chronic low-grade inflammatory diseases such as insulin resistance atherosclerosis [12]. Thus, therapeutics aimed at activating AMPK pathway unravels a promising approach in obesity and metabolic dysfunction.

Alpha-Lipoic acid (ALA) is a naturally occurring antioxidant which acts as a cofactor of mitochondrial respiratory chain enzymes and plays a crucial role in Krebs cycle phase of the mitochondrial energy metabolism [13]. ALA dose-dependently reduced body weight by increasing energy expenditure accompanied with enhanced Ucp 1 mRNA expression in BAT, which might result from the suppression of hypothalamic AMPK activity [14].Recent investigation suggests that ALA induced mitochondria biogenesis and promoted browning of WAT in human subcutaneous adipocytes [15] ; however, its effect in vivo has not been fully established. The aim of the present study is to investigate the anti-obesity effects of ALA in Ovx rats and whether AMPK is involved in the browning capacities.

\section{Materials And Methods}

\section{Animal preparation}

Female Wistar rats (8 weeks old, 250-270 g) were obtained from BioLASCO Taiwan Co., Ltd., Taiwan. Animal Handling was in accordance with the Guide for the Care and Use of Laboratory Animals published by the US National Institutes of Health (NIH Publication No. 85 - 23, revised in 1996). This study was approved by the Institutional Animal Care and Use Committee of the National Defense Medical Center, Taiwan (Permission No. IACUC-16-089). To induce estrogen-insufficiency condition, rats were anesthetized with sodium pentobarbital ( $50 \mathrm{mg} / \mathrm{kg}$, intraperitoneal injection) and subjected to bilateral ovariectomy. Small incisions were proceeded bilaterally on the back sides to expose the retroperitoneal ovaries. The ovaries were clamped and discarded, and the uterine tubes were ligated, following the suture 
of muscle and skin. The procedure of sham operation consisted of anesthesia, visualization of the ovaries through incisions into the abdominal cavity, and closure of the wounds as described previously [16].

\section{Experimental groups}

Rats were randomly divided into four groups: (I) Sham: rats were subjected to sham operations $(\mathrm{n}=8)$; (II) Ovx: rats were ovariectomized bilaterally $(n=11)$; (III) Ovx + ALA2: Ovx rats were administered with ALA (200 mg/kg/day, gavage) (Sigma-Aldrich, St. Louis, MO, USA) beginning 1 week after operation $(n=10)$; (IV) Ovx + ALA3: Ovx rats were administrated with ALA (300 mg/kg/day, gavage) beginning 1 week after operation $(n=6)$. After 8 weeks of treatment, rats were then sacrificed and adipose tissues were dissected, weighed, immediately frozen in liquid nitrogen and stored at $-80^{\circ} \mathrm{C}$ for further analysis.

\section{Measurement of serum lipid profiles}

Whole blood samples $(1 \mathrm{~mL})$ were collected by cardiac puncture and centrifuged at $12,000 \mathrm{~g}$ for 5 minutes at $4{ }^{\circ} \mathrm{C}$. Serum triglycerides (TG), cholesterol (TC), low-density lipoprotein cholesterol (LDL-C), and high-density lipoprotein cholesterol (HDL-C) were detected by a Fuji DRI-CHEM 3030 analyzer (Fuji Photo Film, Tokyo, Japan).

\section{Enzyme-linked immunosorbent assay (ELISA)}

Determination of serum irisin levels were performed using commercial ELISA kit (BioVision Inc., Milpitas, CA, USA), according to the manufacture's instruction.

\section{Histological analysis and immunohistochemistry (IHC) staining}

Inguinal WAT was fixed in $10 \%$ paraformaldehyde, embedded in paraffin blocks, and serial sections were stained with Hematoxylin \& Eosin (H\&E) for histological analysis. For IHC stain, sections of inguinal adipose tissue were deparaffinized, boiled in antigen-retrieval solution, and treated with anti-UCP1 antibody (Abcam, Cambridge, MA, USA) at a dilution of 1:500.

\section{Western blot analysis}

Proteins were separated by sodium dodecyl sulfate-polyacrylamide gel electrophoresis (SDS-PAGE) and transferred to nitrocellulose membranes. The membranes were blocked with $5 \%$ bovine serum albumin in Tris-buffered saline containing $0.1 \%$ Tween $20 \%$ (TBST) for $1 \mathrm{hr}$ at room temperature. Primary antibodies against p-AMPK, AMPK, p-ACC, ACC, LKB1 1:1000) and $\beta$-actin (1:5000, all Cell Signaling Technology, Danvers, MA, USA); UCP1, PRDM16, CIDEA (1:1000, all Abcam, Cambridge, MA, USA) were incubate at 4 ${ }^{\circ} \mathrm{C}$ overnight. The membranes were then probed with corresponding secondary antibodies conjugated with HRP (1:3000, Cell Signaling Technology). The density of the individual protein bands was quantified by densitometric scanning using ImageJ software.

\section{Statistical analysis}


These data are presented as means \pm standard errors of the mean (SEM). A Two-Way ANOVA and LSD post-hoc comparison test was used to compare the means of body weight and food intake between the groups and time. Statistical evaluation of others was performed with one-way analysis of variance (ANOVA) followed by the Newman-Keuls comparison method. Differences with $P<0.05$ was considered as statistical significance.

\section{Results}

\section{Effects of ALA on body weight (BW) and food intake in Ovx rats}

As shown in Fig. 1A, nine weeks after Ovx, body weight (BW) of Ovx (374.3 $\pm 8.2 \mathrm{~g})$ group was significantly increased as compared to Sham group $(296.6 \pm 1.21 \mathrm{~g})(\mathrm{P}<0.05)$. Ovx + ALA2 $(333.1 \pm 7.4 \mathrm{~g})$ and Ovx + ALA3 $(309.3 \pm 3.7 \mathrm{~g})$ groups showed significantly lower levels of BW than that of Ovx group (P $<0.05)$. As shown in Fig. 1B, there was no significant difference in food intake among the groups at the time of 8- week treatment.

\section{Effects of ALA on visceral and inguinal fat pad mass in Ovx rats}

Total visceral fat pad mass was consisted of peri-renal, retroperitoneal and mesentery adipose tissue weight. Figure $1 \mathrm{C}$ showed Ovx groups $(19.0 \pm 1.1 \mathrm{~g})$ exhibited significant increases in total visceral fat pad mass when compared with the Sham group $(11.2 \pm 1.4 \mathrm{~g})(P<0.05)$. The increase of visceral fat pad mass in Ovx rats was significantly reduced in Ovx + ALA2 $(16.0 \pm 1.7 \mathrm{~g})$ and Ovx + ALA3 $(7.9 \pm 0.6 \mathrm{~g})(\mathrm{P}<$ 0.05). Similar results were observed in the inguinal fat pat mass, showing that ALA treatment drastically attenuated inguinal fat pad mass in a dose dependent manner (Fig. 1D).

\section{ALA treatment improved lipid profiles of TG, TC, LDL-C, and HDL-C in Ovx rats}

We next evaluated the effects of ALA on lipid profiles of Ovx rats. As shown in Fig. 2A, serum levels of triglycerides $(T G)$ in the Ovx group were significantly increased when compared to Sham group $(P<0.05)$. High dose ALA treatment $(300 \mathrm{mg} / \mathrm{kg}$ ) markedly reduced the triglyceride levels as compared to the Ovx group $(P<0.05)$ Moreover, ALA supplement decreased Ovx-induced elevation of serum levels of cholesterol $(T C)$ and LDL-C $(P<0.05)$ (Fig. 2B and $C)$. The 8-week ALA treatment in Ovx rats resulted in a significant increase in serum HDL-C levels $(P<0.05)$ (Fig. 2D).

Effects of ALA treatment on H\&E staining for adipocyte sizes and immunohistochemistry for UCP1 expression in inguinal WAT in Ovx rats

Adipocyte sizes of inguinal white adipose tissue (WAT) were assessed by H\&E stain to examine the morphology changes. As shown in Fig. 3A \& B, adipocyte sizes in the Ovx group were significantly greater 
than those of Sham group, which were dramatically diminished in the Ovx + ALA2 and Ovx + ALA3 groups $(P<0.05)$.

Meanwhile, by immunohistochemical (IHC) staining of UCP1, we found significant increase of UCP1positive stained area was observed in the Ovx + ALA2 and Ovx + ALA3 groups as compared with the Ovx group $(P<0.05)$ (Fig. 3C\&D), suggesting that the thermogenic small multilocular beige adipocytes formation was triggered by ALA treatment.

ALA increased brown fat-regulated proteins expression in inguinal WAT of Ovx rats

Consistent with IHC results, Western blot analysis in inguinal WAT showed that UCP1 protein expression was significantly lower in Ovx group than that of Sham group (Fig. 4A). ALA treatment significantly upregulated the protein expression of UCP1 as compared to Ovx group $(P<0.05)$. In addition, transcription factors that regulate the trans-differentiation of white into beige adipocyte including PR domain containing 16 (PRDM16) (Fig. 4B), and cell death-inducing DNA fragmentation factor alpha-like effector A (CIDEA) (Fig. 4C) were significantly upregulated in Ovx + ALA2 and Ovx + ALA3 groups. Collectively, these results indicated ALAL induced browning of inguinal WAT.

\section{Effects of ALA on serum irisin levels in Ovx rats}

Irisin is an exercise-induced myokine secreted from skeletal muscle and associated with enhanced energy expenditure because of the ability to induce WAT browning [17]. We then determined whether ALA treatment stimulated the circulating levels of irisin in Ovx rats. As shown in Fig. 5, serum irisin levels were significantly reduced in the Ovx group as compared with the Sham group $(P<0.05)$, an effect that was drastically reversed by ALA treatment.

\section{ALA induces the activation of AMPK pathway in inguinal WAT of Ovx rats}

AMPK activation is a master energy switch regulator and plays an important role in mediating the browning of WAT [18], we then measured changes in phosphorylation levels of AMPK in Ovx rats. As shown in Fig. 6A, the expression of p-AMPK/AMPK was significantly lower in Ovx group than that of Sham group. ALA supplementation markedly increased the $\mathrm{p}$-AMPK/AMPK levels in Ovx rats $(P<0.05)$. AMPK is mainly activated by phosphorylation of the a subunit at Thr172 by liver kinase B1 (LKB1) under energy stress [10]. We found that ALA markedly increased the expression LKB1 in Ovx rats (Fig. 6B). AMPK activation phosphorylates and inhibits acetyl-CoA carboxylase (ACC), an important enzyme in promoting fatty acid oxidation and reduces fat accumulation. Figure $6 \mathrm{C}$ indicated $\mathrm{p}-\mathrm{ACC} / \mathrm{ACC}$ expression was dramatically increased by ALA administration in Ovx rats. These results suggested that LKB1-AMPKACC signaling is involved in the browning effect of ALA on iWAT.

\section{Discussion}

In the present study, we showed that administration of ALA significantly suppressed the elevation in body weight and fat pad mass after ovariectomy. We further indicated that serum concentration of TG, TC and 
LDL-C were higher and that of HDL-C was lower in Ovx rats, but these deleterious effects were ameliorated by ALA supplementation. In addition, ALA treatment reduced adipocyte size and exhibited unique staining of UCP1 in inguinal WAT. Myokine irisin secretion as well as expression of thermogenic proteins such as UCP1, PRDM16 and CIDEA were upregulated by ALA, suggesting that ALA exerts the browning capacities in Ovx rats. We further demonstrated that ALA treatment increased LKB1 and stimulated the phosphorylation of AMPK-Thr ${ }^{172}$ and its downstream target ACC, revealing the beneficial effects are mediated at least in part by activation of the LKB1/AMPK pathway. To the best of our knowledge, this is the first study to examine the effects of ALA on estrogen-deficiency induced metabolic alterations and WAT browning via activation of LKB1/AMPK.

Several studies have addressed that oxidative stress during menopause might aggravate the inflammatory state associated with obesity and metabolic syndromes [19]. Estrogen exerts antioxidant and anti-inflammatory effects in modulation insulin action [20]. Moreover, reduced energy expenditure during menopause transition may predispose to obesity and dyslipidemia [21]. Thus, antioxidant treatment has been proposed as a therapy strategy to prevent obesity and associated comorbidities. In line with our report, Delgobo et al., showed ALA improved antioxidant defenses and alleviated oxidative stress, inflammation and serum lipid in estrogen-independent mechanism [22]. The enhancement of energy expenditure in BAT or inducing the trans-differentiation of white to beige adipocytes because these strategies have the potential to prevent the metabolic complications of obesity [23]. Development of beige adipocytes in WAT enhances energy expenditure and switches adipocytes from an energy storage state to an energy dissipation state via expressing UCP1 [6], which reduces the risk of obesity and metabolic diseases. We demonstrated that major transcriptional regulators of WAT browning such as PRDM16, PGC-1 $a$ and CIDEA were upregulated by ALA (Fig. 4). PGC1 a promotes UCP1 to activate thermogenesis in these two distinct types of adipocytes [46], while PRDM16 plays a critical role during the differentiation of BAT and trans-differentiation of white into beige adipocytes. Furthermore, irisin is secreted as a hormone from muscle into the circulation following cleavage of fibronectin type 3 domain containing protein 5 (FNDC5) to induce browning of WAT [24]. Emerging evidence supports that irisin has little effect on classical BAT isolated from the interscapular depot, suggesting that the activation of the thermogenic program in response to irisin is a selective characteristic of beige cells [25]. We found that Ovx decreased browning of iWAT and irisin secretion, leading to weight gain after estrogen depletion (Fig. 5), this finding is in line with our previous literature [7]. Sul et al further indicated exogenous administration of $\mathrm{E}_{2}$ enhanced browning in vivo and invitro, which might result from the anti-oxidant activity of $E_{2}$ [26]. ALA supplementation increased the serum levels of irisin, suggesting the browning effects of ALA is partly mediated via irisin production. The lower weight gain in ALA-treated Ovx rats was in accordance with previous report, which showed the anti-obesity effects by ALA is due to enhancement of energy expenditure [14]. ALA supplementation displayed the reduction of fat depots and appearance of multiocular adipocytes within the iWAT, which may be attributed to an increase in lipolysis [27] and decrease in adipogenesis [28]. 
Estrogen deficiency-induced augmentation of oxidative stress alters lipid metabolism, resulting in excessive TG, TC, and LDL-C accumulation, which is a common risk factor for the development metabolic dysfunction $[29,30]$. The current study showed ALA treatment reduced TG, TC, and LDL-C as well as increased HDL-C levels in Ovx rats (Fig. 2). These results are in accordance with previous report showing that ALA reduced TG accumulation in skeletal muscles, pancreatic islets, and adipose tissue in diabetesprone obese rats and increased fatty acid oxidation [31]. Evidence has also been shown that the improvement in lipid profile of ALA may be associated with the antioxidative and anti-inflammatory properties [32]. Moreover, BAT and beige adipocytes generate heat by utilizing TG-derived fatty acids from the circulation in a UCP1-dependent process, leading to the improvement of lipid profiles and prevention of atherosclerosis progression [33]. Thus, the beneficial effects of ALA in lowering TG and cholesterol might act as a consequence of the induction of browning in inguinal WAT.

Activation of AMPK inhibits fatty acid synthesis and enhances fatty acid oxidation by phosphorylation of its downstream target ACC [34], which directs FAs towards degradation. Previous studies showed that reduction of PAMPK in WAT is widely observed in obese and diabetic rodents as well as in human subjects accompanied with insulin resistance [35]. In addition, deletion of AMPKa1 impaired the thermogenic program in BAT [36], which may result from the defective unwinding of the DNA of the promoter region of the PRDM16 [37]. These data suggest that activation of AMPK plays an important role in thermogenic function. In the present study, we showed that Ovx rats have reduction at several steps of the AMPK pathway, spanning from the upstream LKB1 to the downstream ACC whereas ALA treatment reversed these deleterious changes during estrogen deficiency (Fig. 6). ALA promoted beige adipocytes development in rats with Ovx and activation of AMPK signaling plays an essential role in mediating the browning effect of ALA.

\section{Conclusion}

In conclusion, as shown in Fig. 7, our present study indicated that ALA effectively reduced $E_{2}$ deficiencyinduced obesity via reprogramming of white to theromogenic beige adipocytes concomitant with the activation of AMPK signaling. Thus, ALA provides a potential preventive strategy for improving postmenopausal obesity.

\section{List Of Abbreviations}

ACC: acetyl-CoA carboxylase

ALA: a-lipoic acid; AMP-activated protein kinase

BAT: brown adipose tissue

CIDEA: cell death-inducing DNA fragmentation factor alpha-like effector $A$

FNDC5: fibronectin type 3 domain containing protein 5 
HDL-C: high density lipoprotein cholesterol; LKB1: liver kinase B1

LDL-C: low-density lipoprotein cholesterol

Ovx: ovariectomized

TC: total cholesterol

TG: triglycerides

PRDM16: PR domain containing 16

UCP1: uncoupling protein 1

WAT: white adipose tissue

\section{Declarations}

Ethics approval and consent to participate: Animal Handling was in accordance with the Guide for the Care and Use of Laboratory Animals published by the US National Institutes of Health (NIH Publication No. 85-23, revised in 1996). This study was approved by the Institutional Animal Care and Use Committee of the National Defense Medical Center, Taiwan (Permission No. IACUC-16-089).

Consent for publication: Not applicable

Availability of data and materials: The datasets generated and/or analyzed during the current study are available in the figshare repository, 10.6084/m9.figshare.14233997. The datasets used and/or analyzed during the current study are available from the corresponding author on reasonable request.

Competing interests: The authors declare that they have no competing interests.

Funding: This work was supported by research grants from the Ministry of National Defense (MAB-109006 and 007), Kaohsiung Armed Forces General Hospital (109-051) and Chi-Mei Hospital (CMNDMC10910), Republic of China, Taiwan.

\section{Authors' contributions:}

M.T.C and S.Y.H contributed equally to all the work for data generation, analysis, interpretation and manuscript preparation. C.W.K, S.Y.C, and P.Y.C performed statistical analysis and generated figures. Y.W.C performed animal experiments and data analysis. H.H.S and Y.M.L generated scientific ideas, study design, data analysis, interpretation, and manuscript writing. All authors read and approved the final manuscript.

Acknowledgements: Not applicable. 


\section{References}

1. Stefanska A, Bergmann K, Sypniewska G. Metabolic Syndrome and Menopause: Pathophysiology, Clinical and Diagnostic Significance. Adv Clin Chem. 2015;72:1-75.

2. Carr MC. The emergence of the metabolic syndrome with menopause. J Clin Endocrinol Metab. 2003;88:2404-11.

3. Stubbins RE, Holcomb VB, Hong J, Nunez NP. Estrogen modulates abdominal adiposity and protects female mice from obesity and impaired glucose tolerance. Eur J Nutr. 2012;51:861-70.

4. Mastorakos G, Valsamakis G, Paltoglou G, Creatsas G. Management of obesity in menopause: diet, exercise, pharmacotherapy and bariatric surgery. Maturitas. 2010;65:219-24.

5. Giralt M, Villarroya F. White, brown, beige/brite: different adipose cells for different functions? Endocrinology. 2013;154:2992-3000.

6. Harms M, Seale P. Brown and beige fat: development, function and therapeutic potential. Nat Med. 2013;19:1252-63.

7. Shen H-H, Huang S-Y, Kung C-W, Chen S-Y, Chen Y-F, et al: Genistein ameliorated obesity accompanied with adipose tissue browning and attenuation of hepatic lipogenesis in ovariectomized rats with high fat diet. J Nutr Biochem 2019.

8. Lee K, Jin H, Chei S, Lee JY, Oh HJ, et al: Dietary Silk Peptide Prevents High-Fat Diet-Induced Obesity and Promotes Adipose Browning by Activating AMP-Activated Protein Kinase in Mice. Nutrients 2020, 12.

9. Hardie DG. AMPK: a key regulator of energy balance in the single cell and the whole organism. Int $J$ Obes (Lond). 2008;32(Suppl 4):7-12.

10. Woods A, Johnstone SR, Dickerson K, Leiper FC, Fryer LG, et al. LKB1 is the upstream kinase in the AMP-activated protein kinase cascade. Curr Biol. 2003;13:2004-8.

11. Fullerton MD, Galic S, Marcinko K, Sikkema S, Pulinilkunnil T, et al. Single phosphorylation sites in Acc1 and Acc2 regulate lipid homeostasis and the insulin-sensitizing effects of metformin. Nat Med. 2013;19:1649-54.

12. Day EA, Ford RJ, Steinberg GR. AMPK as a Therapeutic Target for Treating Metabolic Diseases. Trends Endocrinol Metab. 2017;28:545-60.

13. Li N, Yan W, Hu X, Huang Y, Wang F, et al. Effects of oral alpha-lipoic acid administration on body weight in overweight or obese subjects: a crossover randomized, double-blind, placebo-controlled trial. Clin Endocrinol (Oxf). 2017;86:680-7.

14. Kim MS, Park JY, Namkoong C, Jang PG, Ryu JW, et al. Anti-obesity effects of alpha-lipoic acid mediated by suppression of hypothalamic AMP-activated protein kinase. Nat Med. 2004;10:727-33.

15. Fernandez-Galilea M, Perez-Matute P, Prieto-Hontoria PL, Houssier M, Burrell MA, et al. alpha-Lipoic acid treatment increases mitochondrial biogenesis and promotes beige adipose features in subcutaneous adipocytes from overweight/obese subjects. Biochim Biophys Acta. 2015;1851:27381. 
16. Shen HH, Yang CY, Kung CW, Chen SY, Wu HM, et al. Raloxifene inhibits adipose tissue inflammation and adipogenesis through Wnt regulation in ovariectomized rats and 3 T3-L1 cells. J Biomed Sci. 2019;26:62.

17. Bostrom P, Wu J, Jedrychowski MP, Korde A, Ye L, et al. A PGC1-alpha-dependent myokine that drives brown-fat-like development of white fat and thermogenesis. Nature. 2012;481:463-8.

18. O'Neill HM, Holloway GP, Steinberg GR. AMPK regulation of fatty acid metabolism and mitochondrial biogenesis: implications for obesity. Mol Cell Endocrinol. 2013;366:135-51.

19. Lizcano F, Guzman G. Estrogen Deficiency and the Origin of Obesity during Menopause. Biomed Res Int 2014, 2014:757461.

20. Ribas V, Nguyen MT, Henstridge DC, Nguyen AK, Beaven SW, et al. Impaired oxidative metabolism and inflammation are associated with insulin resistance in ERalpha-deficient mice. Am J Physiol Endocrinol Metab. 2010;298::E304-19.

21. Lovejoy JC, Champagne CM, de Jonge L, Xie H, Smith SR. Increased visceral fat and decreased energy expenditure during the menopausal transition. Int J Obes (Lond). 2008;32:949-58.

22. Delgobo M, Agnes JP, Gonçalves RM, Dos Santos VW, Parisotto EB, et al. N-acetylcysteine and alphalipoic acid improve antioxidant defenses and decrease oxidative stress, inflammation and serum lipid levels in ovariectomized rats via estrogen-independent mechanisms. J Nutr Biochem. 2019;67:190-200.

23. Bartelt A, Heeren J. Adipose tissue browning and metabolic health. Nat Rev Endocrinol. 2014;10:2436.

24. Zhang Y, Li R, Meng Y, Li S, Donelan W, et al. Irisin stimulates browning of white adipocytes through mitogen-activated protein kinase p38 MAP kinase and ERK MAP kinase signaling. Diabetes. 2014;63:514-25.

25. Wu J, Boström P, Sparks LM, Ye L, Choi JH, et al. Beige adipocytes are a distinct type of thermogenic fat cell in mouse and human. Cell. 2012;150:366-76.

26. Sul OJ, Hyun HJ, Rajasekaran M, Suh JH, Choi HS. Estrogen enhances browning in adipose tissue by M2 macrophage polarization via heme oxygenase-1. J Cell Physiol. 2021;236:1875-88.

27. Fernandez-Galilea M, Perez-Matute P, Prieto-Hontoria PL, Martinez JA, Moreno-Aliaga MJ. Effects of lipoic acid on lipolysis in 3T3-L1 adipocytes. J Lipid Res. 2012;53:2296-306.

28. Hahm JR, Noh HS, Ha JH, Roh GS, Kim DR. Alpha-lipoic acid attenuates adipocyte differentiation and lipid accumulation in 3T3-L1 cells via AMPK-dependent autophagy. Life Sci. 2014;100:125-32.

29. Signorelli SS, Neri S, Sciacchitano S, Pino LD, Costa MP, et al. Behaviour of some indicators of oxidative stress in postmenopausal and fertile women. Maturitas. 2006;53:77-82.

30. Creatsas G, Christodoulakos G, Lambrinoudaki I. Cardiovascular disease: screening and management of the a-symptomatic high-risk post-menopausal woman. Maturitas. 2005;52(Suppl 1):32-7. 
31. Lee WJ, Song KH, Koh EH, Won JC, Kim HS, et al. Alpha-lipoic acid increases insulin sensitivity by activating AMPK in skeletal muscle. Biochem Biophys Res Commun. 2005;332:885-91.

32. Zhang Y, Han P, Wu N, He B, Lu Y, et al. Amelioration of lipid abnormalities by a-lipoic acid through antioxidative and anti-inflammatory effects. Obesity (Silver Spring). 2011;19:1647-53.

33. Khedoe PP, Hoeke G, Kooijman S, Dijk W, Buijs JT, et al. Brown adipose tissue takes up plasma triglycerides mostly after lipolysis. J Lipid Res. 2015;56:51-9.

34. Janovska A, Hatzinikolas G, Staikopoulos V, Mclnerney J, Mano M, et al. AMPK and ACC phosphorylation: effect of leptin, muscle fibre type and obesity. Mol Cell Endocrinol. 2008;284:1-10.

35. Xu XJ, Gauthier MS, Hess DT, Apovian CM, Cacicedo JM, et al. Insulin sensitive and resistant obesity in humans: AMPK activity, oxidative stress, and depot-specific changes in gene expression in adipose tissue. J Lipid Res. 2012;53:792-801.

36. Zhao J, Yang Q, Zhang L, Liang X, Sun X, et al. AMPKa1 deficiency suppresses brown adipogenesis in favor of fibrogenesis during brown adipose tissue development. Biochem Biophys Res Commun. 2017;491:508-14.

37. Yang Q, Liang X, Sun X, Zhang L, Fu X, et al. AMPK/a-Ketoglutarate Axis Dynamically Mediates DNA Demethylation in the Prdm16 Promoter and Brown Adipogenesis. Cell Metab. 2016;24:542-54.

\section{Figures}


Fig.1

A

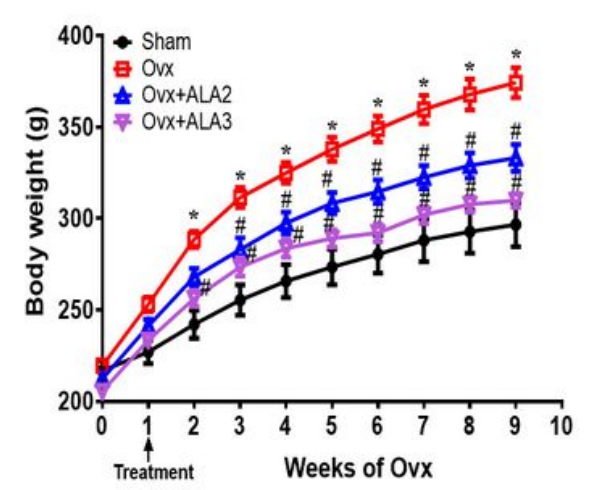

B
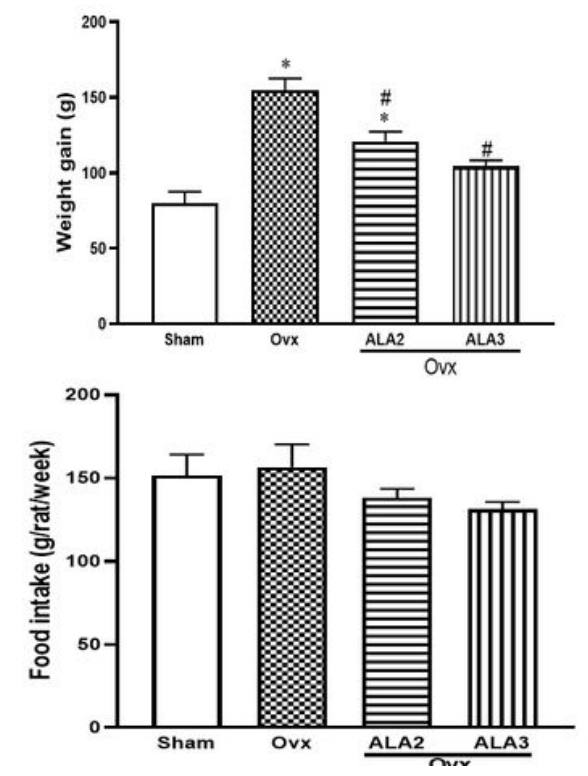

Fig.1

C

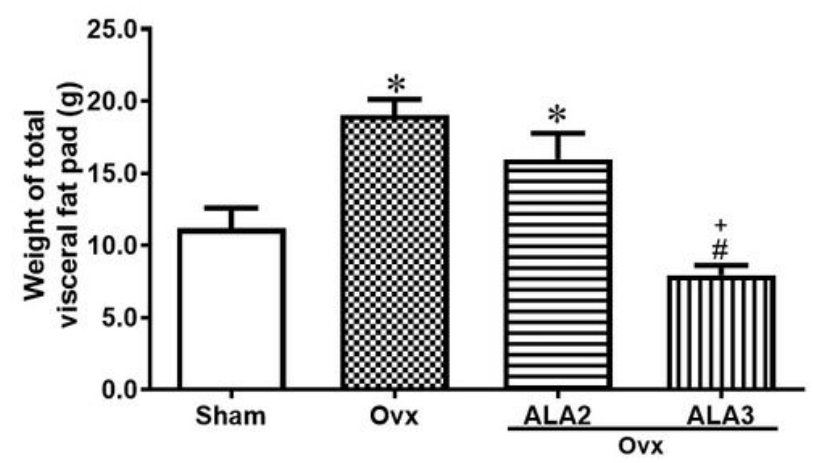

D

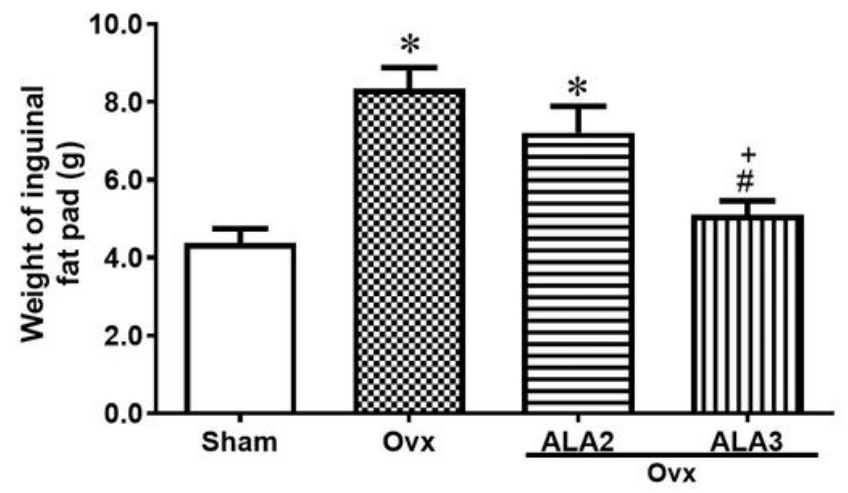

Figure 1

Effects of treatment with a-lipoic acid (ALA) on body weight and fat pad mass in ovariectomized rats. (A) time-course changes of body weight and body weight gain from 0 to 9 weeks, $(B)$ food intake, (C) total visceral and (D) inguinal fat pad mass were measured after ALA treatment for 8 weeks. Data are expressed as mean \pm SEM. ${ }^{*} P<0.05$ versus Sham; $\# P<0.05$ versus $0 v x ;+P<0.05$ versus $0 v x+A L A 2$. $n=$ 8-11. Ovx: ovariectomy; ALA2: a-lipoic acid (200 mg/kg/day, gavage); ALA3: a-lipoic acid (300 $\mathrm{mg} / \mathrm{kg} /$ day, gavage) for 8 weeks. 
Fig. 2

A

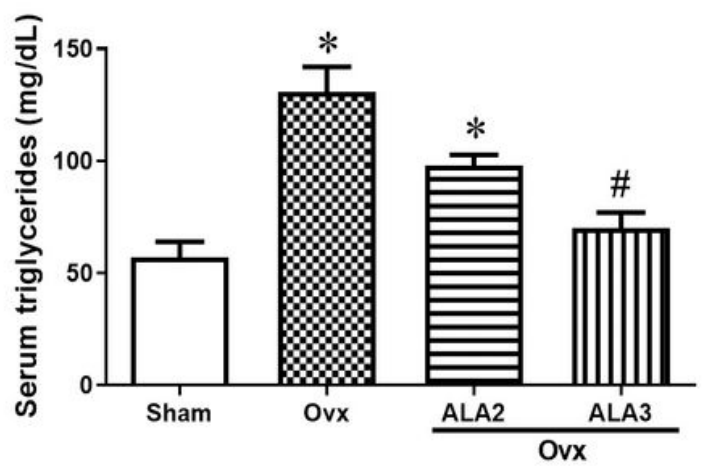

B

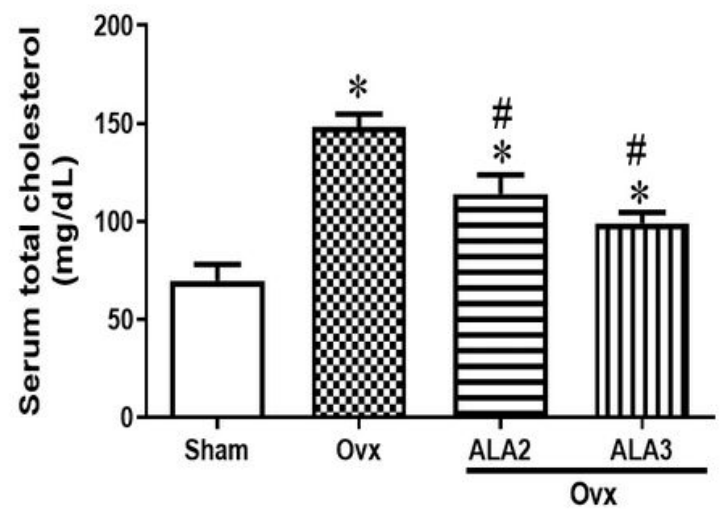

Fig.2

C
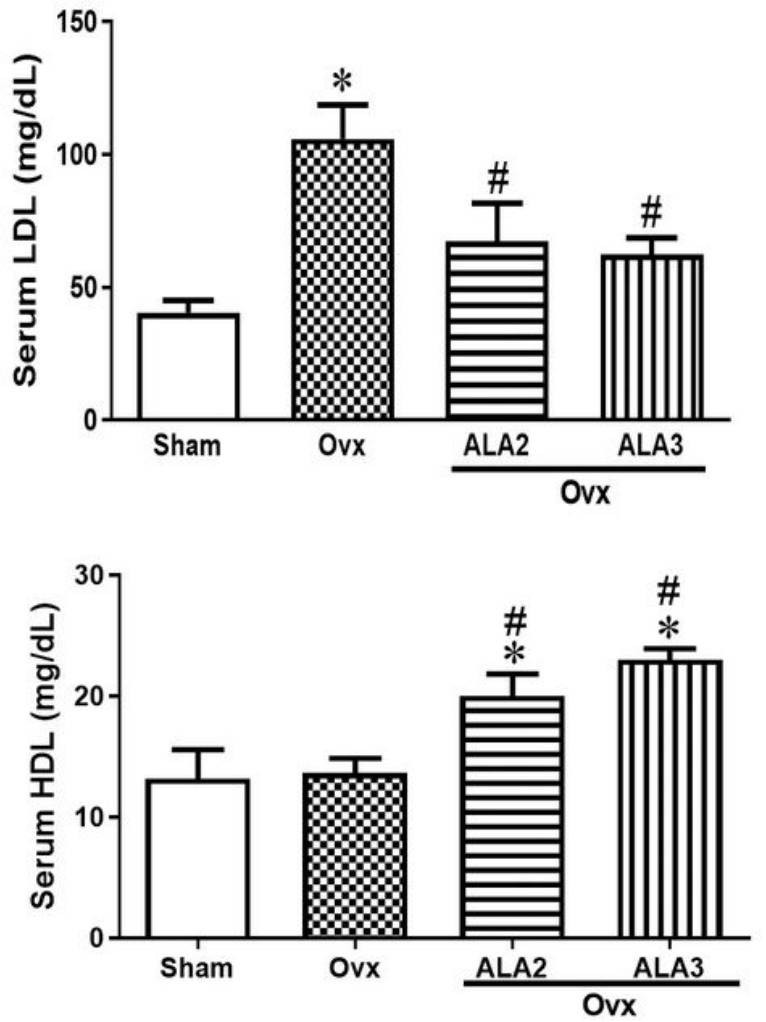

Figure 2

Effects of treatment with a-lipoic acid (ALA) on serum levels of (A) triglycerides, (B) total cholesterol, (C) low density lipoprotein cholesterol (LDL-C) and (D) high density lipoprotein cholesterol (HDL-C) in ovariectomized rats. Data are expressed as mean \pm SEM. ${ }^{*} P<0.05$ versus Sham; $\# P<0.05$ versus Ovx. $\mathrm{n}=8-11$. Ovx: ovariectomy; ALA2: a-lipoic acid treatment $(200 \mathrm{mg} / \mathrm{kg} /$ day, gavage); ALA3: a-lipoic acid (300 mg/kg/day, gavage) for 8 weeks. 


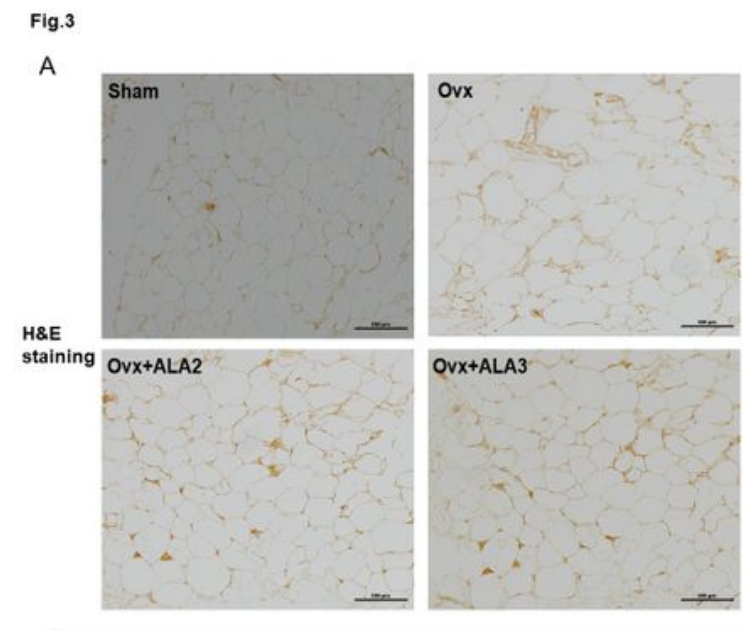

B

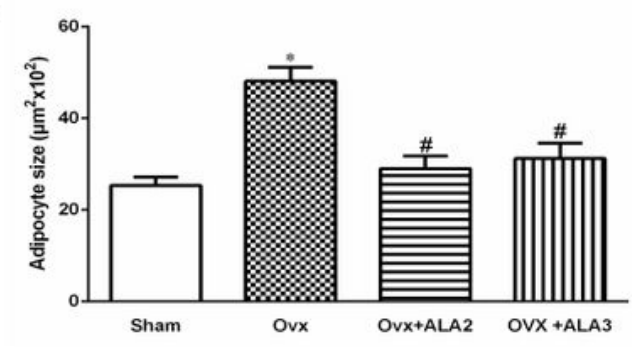

Fig. 3

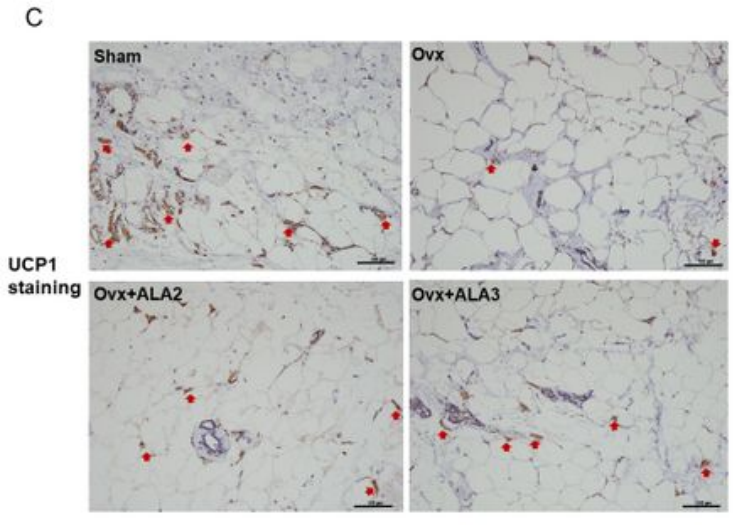

\section{Figure 3}

Effects of treatment with a-lipoic acid (ALA) on adipocyte size and UCP1 expression in inguinal white adipose tissue of ovariectomized rats. (A) Representative photomicrographs of cross sections using Hematoxylin-Eosin and (B) the quantification of adipocyte size; (C) representatives of immunohistochemistry stain for UCP1. The images were captured at 200X magnification. Data are expressed as mean \pm SEM. ${ }^{*} \mathrm{P}<0.05$ versus Sham; $\# \mathrm{P}<0.05$ versus Ovx. $\mathrm{n}=8-11$. Ovx: ovariectomy; 
ALA2: a-lipoic acid treatment (200 mg/kg/day, gavage); ALA3: a-lipoic acid (300 mg/kg/day, gavage) for 8 weeks.

Fig.4

A

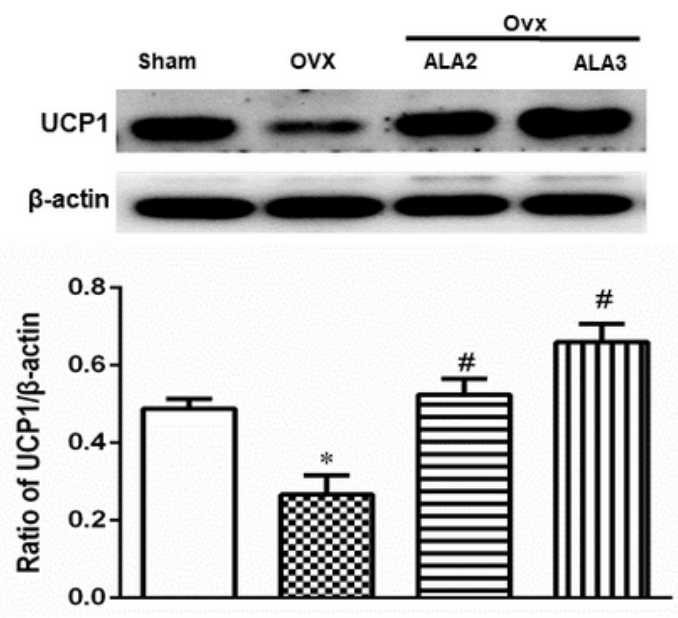

B
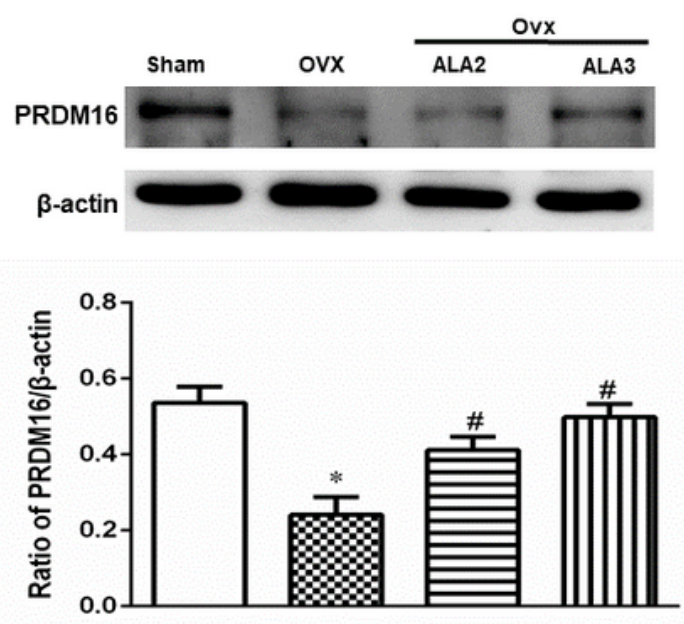

C
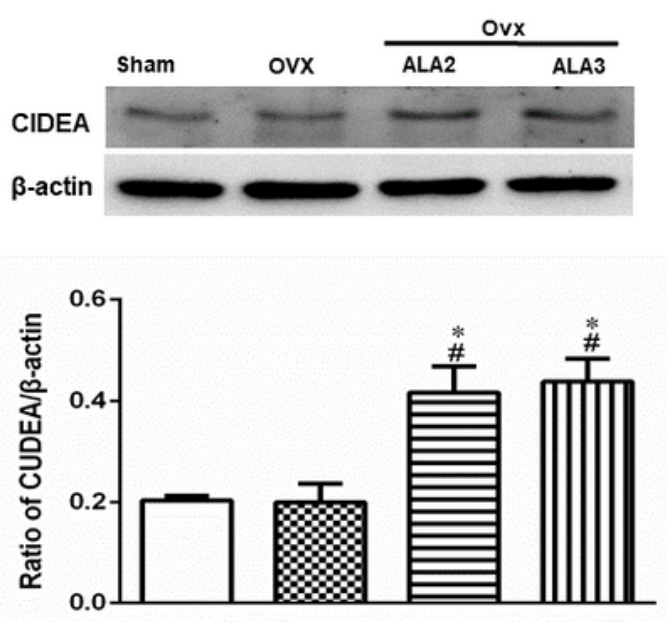

Figure 4

Effects of long-term treatment with raloxifene on the markers of white adipose tissue (WAT) browning in inguinal WAT of ovariectomized rats. Representative protein expression and quantification of (A) UCP1, (B) PRDM16, and (C) CIDEA. Data are expressed as mean \pm SEM. ${ }^{*} P<0.05$ versus Sham; \# $P<0.05$ 
versus Ovx. $\mathrm{n}=8-11$. Ovx: ovariectomy; ALA2: a-lipoic acid treatment (200 mg/kg/day, gavage); ALA3: alipoic acid (300 mg/kg/day, gavage) for 8 weeks.

Fig.5

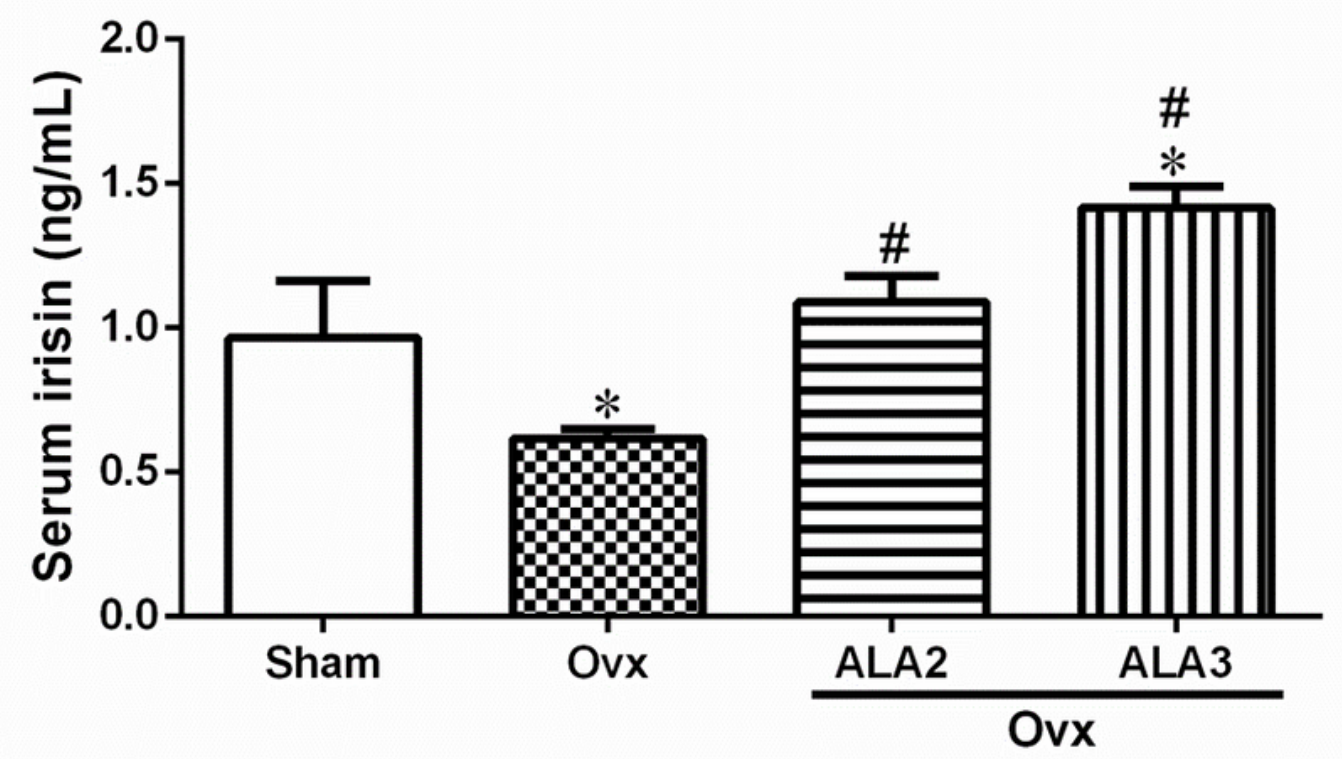

Figure 5

Effects of treatment with a-lipoic acid (ALA) on serum levels of irisin in ovariectomized rats. Data are expressed as mean \pm SEM. ${ }^{*} \mathrm{P}<0.05$ versus Sham; $\# \mathrm{P}<0.05$ versus $0 v x . \mathrm{n}=8-11$. Ovx: ovariectomy; 
ALA2: a-lipoic acid treatment (200 mg/kg/day, gavage); ALA3: a-lipoic acid (300 mg/kg/day, gavage) for 8 weeks.

Fig.6

A
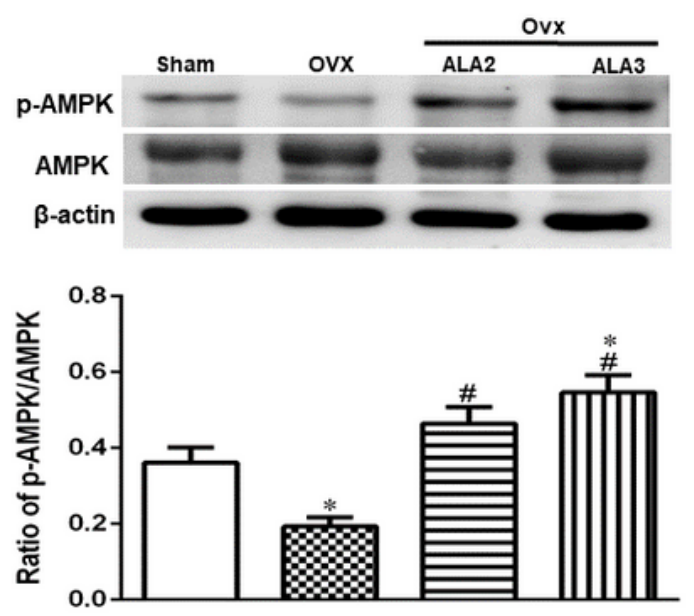

B
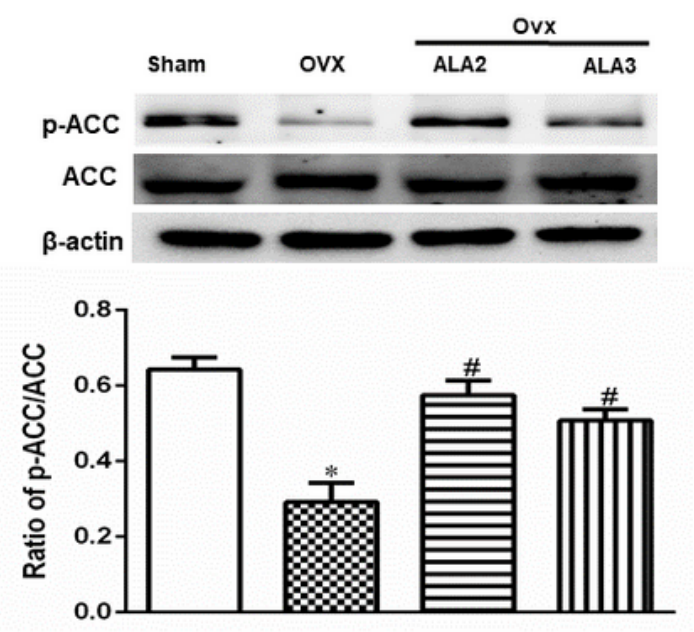

C
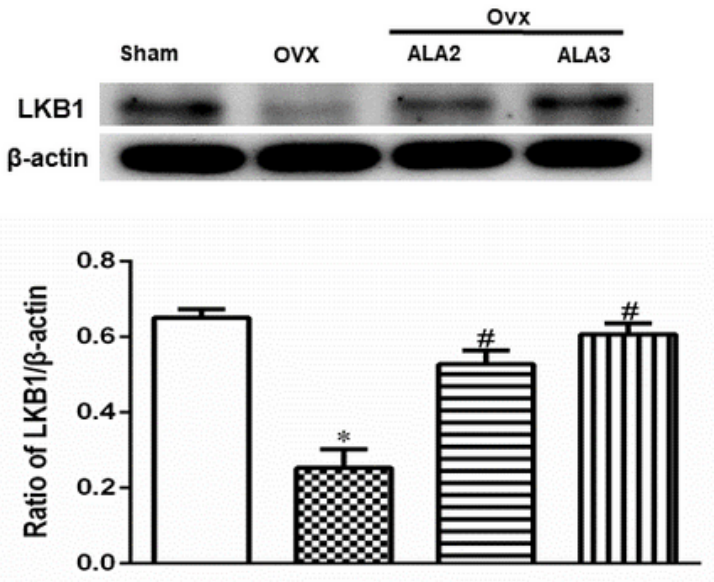

Figure 6

Effects of treatment with a-lipoic acid (ALA) on AMPK signaling in inguinal white adipose tissue (WAT) of ovariectomized rats. Representative protein expression and quantification of (A) pAMPK, (B) pACC, and (C) LKB1. Data are expressed as mean \pm SEM. ${ }^{*} \mathrm{P}<0.05$ versus Sham; $\# \mathrm{P}<0.05$ versus Ovx. $\mathrm{n}=8-11$. 
Ovx: ovariectomy; ALA2: alipoic acid treatment ( $200 \mathrm{mg} / \mathrm{kg} /$ day, gavage); ALA3: a-lipoic acid (300 $\mathrm{mg} / \mathrm{kg} /$ day, gavage) for 8 weeks.

Fig. 7

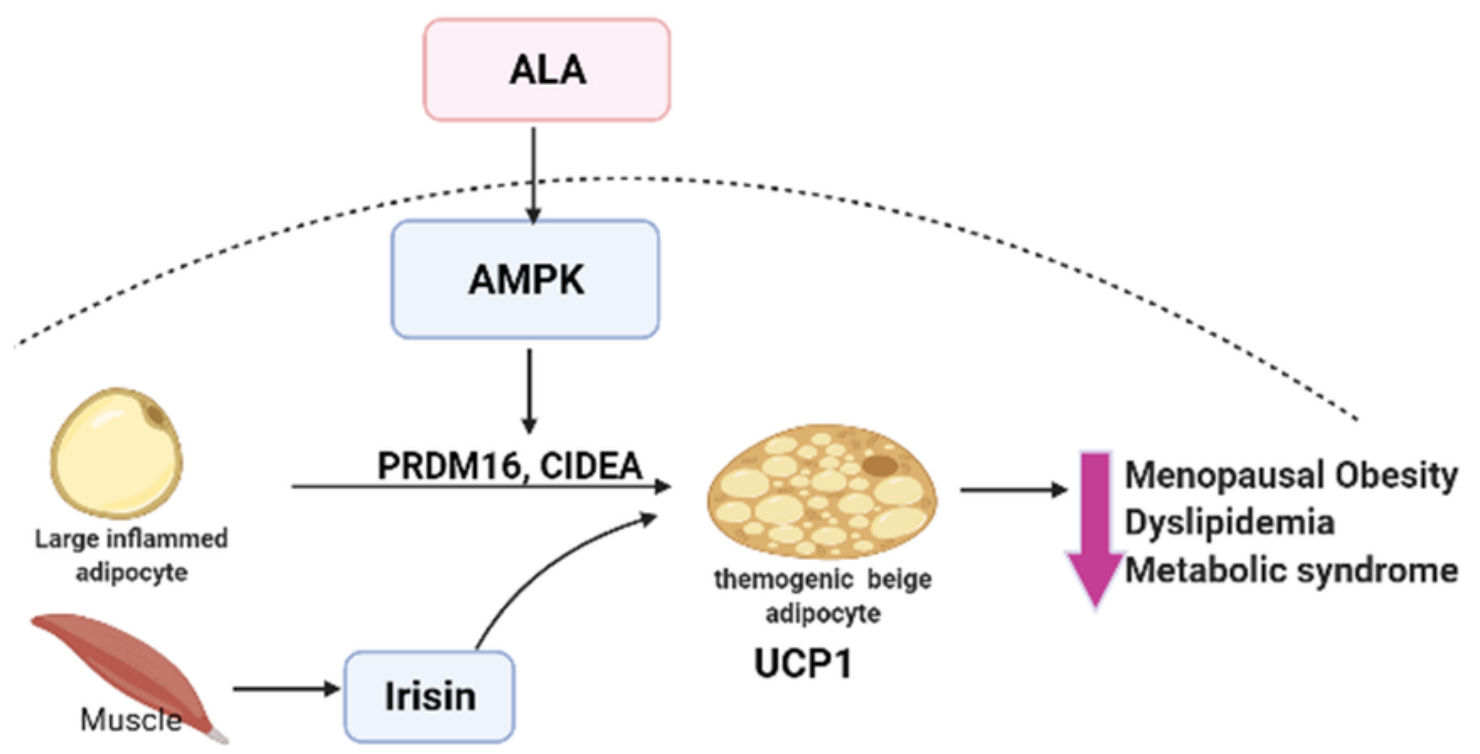

\section{Figure 7}

Schematic depiction showing that a-lipoic acid (ALA) activates AMPK signaling and irisin secretion for the induction of UCP1, PRDM16 and CIDEA and coordinate the conversion of white adipocyte to beige phenotype, leading to the improvement of estrogen deficiency-induced obesity. AMPK: AMP-activated 
protein kinase; CIDEA: cell death-inducing DNA fragmentation factor alpha-like effector A; PRDM16: PR domain containing 16; UCP1: uncoupling protein 1. 